\title{
Psychological flexibility, occupational burnout and eating behavior among working women ${ }^{*}$
}

\author{
Nina Nevanperä1 ${ }^{1 \#}$, Raimo Lappalainen², Eeva Kuosma ${ }^{3}$, Leila Hopsu ${ }^{3}$, Jukka Uitti ${ }^{4}$, \\ Jaana Laitinen ${ }^{1}$ \\ ${ }^{1}$ Finnish Institute of Occupational Health, Oulu, Finland; ${ }^{\#}$ Corresponding Author: nina.nevanpera@,ttl.fi, jaana.laitinen@ttl.fi \\ ${ }^{2}$ University of Jyväskylä, Jyväskylä, Finland; raimo.lappalainen@jyu.fi \\ ${ }^{3}$ Finnish Institute of Occupational Health, Helsinki, Finland; eeva.kuosma@ttl.fi, leila.hopsu@ttl.fi \\ ${ }^{4}$ Finnish Institute of Occupational Health, Tampere, Finland; jukka.uitti@ttl.fi
}

Received 19 April 2013; revised 20 May 2013; accepted 30 May 2013

Copyright (C) 2013 Nina Nevanperä et al. This is an open access article distributed under the Creative Commons Attribution License, which permits unrestricted use, distribution, and reproduction in any medium, provided the original work is properly cited.

\section{ABSTRACT}

Background: Occupational burnout is associated with diminished psychological flexibility and higher emotional (EE) and uncontrolled eating (UE). Psychological flexibility could be a mediating factor between burnout and eating behaviour. Objectives: To investigate differences in eating behaviour between those with different levels of psychological flexibility, and the association of the interaction between psychological flexibility and occupational burnout with eating behaviour. Design: The participants were working women $(n=263)$, who took part in the randomized controlled health intervention trial. Analyses were performed in a cross-sectional setting at baseline. Methods: Eating behaviour was measured using the Three Factor Eating Behaviour Questionnaire-18 [which evaluates EE, UE and cognitive restraint (CR)], psychological flexibility using Acceptance and Action Questionnaire-II and occupational burnout using Bergen Burnout Indicator-15. Participants were divided into four groups based on the quartile points of psychological flexibility. Results: The EE of those who were inflexible was higher than that of those whose flexibility was high moderate $(p=0.013)$ and who were flexible $(p=0.001)$. The UE of those who were inflexible was higher than the UE in the other groups with higher fle-

\footnotetext{
${ }^{*}$ This article was financially supported by the Academy of Finland (129248) and by the SalWe Research Program for Mind and Body (Tekes - the Finnish Funding Agency for Technology and Innovation grant 1104/10). The Nuadu health intervention study was supported by the Finnish Funding Agency for Technology and Innovation (TEKES), the Finnish Institute of Occupational Health (FIOH) and the Technical Research Centre of Finland (VTT).
}

xibility [low moderate $(p=0.034)$, high moderate $(p<0.001)$, and flexible $(p<0.001)$ ]. Psychological flexibility diluted the association between occupational burnout and EE and UE. Multivariate analysis of variances revealed that the combination of psychological flexibility and burnout had a stronger association with the variances of EE and UE than psychological flexibility alone. Conclusions: Persons who are psychologically inflexible have higher EE and UE. Future studies should investigate if increasing psychological flexibility helps decrease EE and UE.

Keywords: Psychological Flexibility; Occupational Burnout; Eating Behavior; Emotional Eating; Uncontrolled Eating; Cognitive Restraint

\section{INTRODUCTION}

In a recent study [1], it was reported that those suffering from occupational burnout have abnormalities in their eating behaviour. Diminished psychological flexibility, also called experiential avoidance, is associated with burnout and characterized by dominance of thoughts, feelings and memories that prevent a person living the life they want to live, and an unwillingness to experience certain negative experiences [2-5]. Burnout is a state that includes prolonged exhaustive fatigue, cynical thoughts and feelings, and impaired occupational self-respect. Psychological flexibility has also been associated with depression, anxiety, stress and job performance amongst other things [4]. Kingston and colleagues (2010) found that experiential avoidance mediated the relationship between negative affect (disposition to experience negative emotional states such as anger, fear and guilt) and 
problem behaviours (including binge eating, smoking and alcohol consumption) among those with psychological problems [6]. Also Ruiz (2010) reported that experiential avoidance mediated relations between different types of symptoms and psychological constructs [7]. Thus, we suggest that psychological flexibility may be a mediator between occupational burnout and eating behaviour.

Abnormal eating behaviour, namely high levels of emotional eating ( $\mathrm{EE}$, i.e. eating due to negative mood states such as sadness and concern) and uncontrolled eating (UE, i.e. not being able to control the amount of food eaten and eating due to external triggers, [8] associate with overweight [9-11] and are related to higher energy and sweet food intake under stress [12]. Decreasing $\mathrm{EE}$ and UE and increasing cognitive restraint, $(\mathrm{CR}$, i.e. restricting eating as an intention to lose or maintain weight) is associated with better results in weight loss interventions [13,14]. However, further knowledge from best practices, and counselling contents and methods are needed in order to develop dietary counselling to better help people with increased EE and UE.

Review made by Barraca (2012) provided promising results for acceptance and mindfulness techniques for coping with intrusive thoughts and emotional response associated with them [15]. Acceptance based interventions aimed at to have an effect on psychological flexibility, emphasizes exposure and acceptance to previously avoided thoughts and feelings. Psychological flexibility is defined as the ability to be in full contact with the present moment, to be aware of and accept thoughts and feelings at the present moment, and at the same time, to act according to one's own values and goals [4]. Diminished psychological flexibility may associate with EE and UE because they may be a way to relieve or escape from difficult thoughts and feelings. Mindfulness has shown to be negatively associated with EE and UE $[16,17]$. Lillis and colleagues (2011) found that reductions in binge eating were mediated by changes in weight-related psychological flexibility [18]. We suggest that EE and UE, which can be triggered by stress or burnout symptoms, may associate with general psychological flexibility.

Therefore, the aim of this study was to examine the differences in EE, UE and CR between those with different levels of psychological flexibility (low, moderate and high) and to investigate whether flexibility is a mediator between burnout and EE and UE, and whether the combination of psychological flexibility and occupational burnout associate with high EE and UE.

\section{METHOD}

\subsection{Participants}

Municipal workers of one of the biggest cities in
Finland $(n=352)$ were recruited to participate in the health intervention study. The aim of the study was to improve the health behaviours of those with health risks such as that of type 2 diabetes, or the risks arising from having two unhealthy lifestyle habits. All municipal workers of the city at issue $(\mathrm{n} \sim 10,900)$ answered the internet-based health questionnaire in the fall of 2007. Of these 4134 answered the questionnaires and 3401 were willing to participate in the study. A total of 783 people were eligible for the study. The baseline data were collected between February and May 2008 from 352 eligible participants who were randomly assigned to the study, were willing to participate in the intervention and to make changes to their health behaviours during the next six months. Study participants had to be aged 30 to 55 , had to rate their work ability between as 7 and 9 on a scale of 0 (completely unable to work) to 10 (work ability at its best), and had to be at risk of type 2 diabetes or have at least two unhealthy behaviours to be eligible for the study.

The detailed study procedures and eligibility criteria concerning health risks and health risk behaviours have been described earlier $[1,19]$. The study was approved by the Ethics Committee of the University Hospital in Finland, and each subject gave written informed consent. Almost half of the participants worked in social and health services, one third in education and culture services, and the rest in technical and environment services or public utility services such as catering.

Analyses of this study were performed in a cross-sectional setting at baseline. Of the 352 participants, men $(n=$ 65 ) were excluded from the analysis because of the statistically significant differences in EE and CR between men and women, and because the number of men was too small number to be able draw any conclusions about results among them [1].

\subsection{Questionnaires}

\subsubsection{Three Factor Eating Behaviour Questionnaire 18 (TFEQ-18)}

The Three Factor Eating Behaviour Questionnaire 18 (TFEQ-18) was used to study EE (i.e., eating due to negative mood states such as sadness and worry), UE (i.e., not being able to control the amount of food eaten and eating due to external triggers) and CR (i.e., restricting eating in order to control body weight [8]. The TFEQ-18 is a new version of the original, 51-item TFEQ. The scores of each eating behaviour trait were summed, and then converted the highest possible sum into percentages.

\subsubsection{Occupational Burnout}

Occupational burnout was evaluated using the Bergen Burnout Indicator 15 (BBI-15) [20]. The measures in- 
clude three characteristics of occupational burnout: 1) exhaustive fatigue; 2) cynicism; and 3) impaired occupational self-respect, and occupational burnout as the sum of these. Exhaustive fatigue means a lack of resources to meet job demands. Cynicism is when a person has lost interest in work and feels that his/her work has no meaning. Impaired occupational self-respect means that fatigue and cynicism extend to structures of ego, and that a person loses his/her self-esteem.

The respondents answered using a Likert-type response scale ranging from 1 (totally disagree) to 6 (totally agree). Total scores summed between 15 and 90 and we adjusted for age and sex. This procedure resulted in four occupational burnout classes according to the percentile of the score: 1) $0 \%$ to $70 \%$, no burnout; 2) $75 \%$ to $80 \%$, mild burnout; 3) $85 \%$ to $90 \%$, moderate burnout; and 4) $95 \%$ to $97.5 \%$, serious burnout. The participants were then further divided into two groups: 1) those without; and 2) those with (mild to serious) burnout.

\subsubsection{Acceptance and Action Questionnaire II (AAQ-II)}

The Finnish version of Acceptance and Action Questionnaire II (AAQ-II) was used to study psychological flexibility. AAQ-II measures psychological inflexibility and experiential avoidance [21]. It is a revised version of the original Acceptance and Action Questionnaire I (AAQ-I), and is a valid, reliable measure of psychological flexibility/inflexibility, acceptance and experiential avoidance [21]. The questionnaire contains ten questions with a Likert-type response scale of scores from 1 to 6 , which diverges from the original version of scores 1 to 7 . The scores of each version were altered to a scale of 1 to 7 to be comparable with the original version. Questions were for example: "I worry about not being able to control my worries and feelings" (revised scale) or "I am in control of my life." The scores of each question were summed so that the sum scores were between 10 and 70 . Low scores indicated psychological inflexibility, and high scores high psychological flexibility.

After this, the scores were divided into groups by quartiles: 1) inflexible (sum scores $\leq 48.4)$; 2) low moderate (48.5 to 55.6$) ; 3$ ) high moderate (55.7 to 61.6$)$; and 4) flexible ( $\geq 61.7)$. In studying the compound association of occupational burnout and psychological flexibility with eating behaviour, the two middle groups were combined into one moderate group.

\subsection{Statistical Analysis}

Statistical analyses were performed using SPSS 17. Women who answered the baseline questionnaire were included in the analysis $(n=263)$. The association between burnout and psychological flexibility was studied using cross-tabulation and the Pearson chi-square test.
Differences in eating behaviour between quartiles of psychological flexibility were studied using the analysis of variance (ANOVA). Next, two models were calculated using multivariate analysis of variances. The first model included psychological flexibility and occupational burnout, in order to investigate whether psychological flexibility affects the association between occupational burnout and eating behaviour (Model 1). After this, the interaction effect of psychological flexibility and occupational burnout was added to the model (Model 2).

\section{RESULTS}

The mean age of the participants at baseline was 44.6 (SD 6.7) years. The mean psychological flexibility score was 54.4 (SD 10.0), the mean CR value was 49.5 (SD 17.6), UE 34.8 (20.1), and EE 44.9 (29.5). Of the participants, 32.5\% were of normal weight (BMI < 25.0), $32.8 \%$ were overweight (BMI 25-29.9) and 34.7\% were obese (BMI $\geq 30.0$ ). Psychological flexibility did not differ between these three weight groups.

\subsection{Eating Behaviour and Psychological Flexibility}

UE $(\mathrm{p}<0.001)$ and EE $(\mathrm{p}<0.001)$ differed according to the levels of psychological flexibility (Table 1). UE was higher among those who were psychologically inflexible than among those with low moderate $(p=0.034)$, or high moderate $(\mathrm{p}<0.001)$ flexibility or those who were flexible $(p<0.001)$. The EE of those who were inflexible was also higher than that of those with high moderate $(\mathrm{p}=0.013)$ or high $(\mathrm{p}<0.001)$ flexibility. The level of psychological flexibility did not affect CR.

\subsection{Psychological Flexibility and Occupational Burnout}

The proportion of those with diminished flexibility was highest among participants suffering from burnout (Table 2, $\mathrm{p}<0.001$ ). Of those with burnout, 50.8\% were inflexible whereas of those without burnout, $18.1 \%$ were inflexible.

\subsection{Eating Behaviour, Psychological Flexibility and Occupational Burnout}

When the associations of occupational burnout and psychological flexibility with UE and EE were studied using multivariate analysis of variance, the association between occupational burnout and UE and EE became diluted and eventually insignificant (Model 1). Multivariate analyses of variance revealed that the interaction of psychological flexibility and occupational burnout had a stronger association with the variances of EE ( $p=$ $0.009)$ and UE $(p=0.001)$ than psychological flexibility 
Table 1. Eating behaviour mean (95\% confidence intervals) by quartile groups of psychological flexibility.

\begin{tabular}{lccccc}
\hline & \multicolumn{4}{c}{ Psychological Flexibility } \\
\cline { 2 - 6 } & $\begin{array}{c}\text { Inflexible } \leq 48.4 \\
\text { points }\end{array}$ & $\begin{array}{c}\text { Low moderate } \\
48.5-55.6\end{array}$ & $\begin{array}{c}\text { High moderate } \\
55.7-61.6\end{array}$ & Flexible $\geq 61.7$ & $\begin{array}{c}\text { Difference between } \\
\text { groups (p) }\end{array}$ \\
\hline $\begin{array}{l}\text { Number of participants } \\
\text { Eating behaviour }\end{array}$ & 67 & 73 & 62 & 61 & \\
Cognitive restraint, CR $(0 \%-100 \%)$ & $48.0(43.8,52.2)$ & $51.7(47.7,55.7)$ & $48.9(44.4,53.4)$ & $48.3(43.5,53.1)$ & $\mathrm{ns}$ \\
Uncontrolled eating, UE $(0 \%-100 \%)$ & $45.0(40.0,50.0)$ & $36.2(31.7,40.6)$ & $30.1(25.6,34.6)$ & $27.8(22.9,32.7)$ & $<0.001^{1}$ \\
Emotional eating, EE $(0 \%-100 \%)$ & $56.2(48.9,63.5)$ & $47.8(41.2,54.4)$ & $40.9(34.1,47.6)$ & $35.5(27.9,43.1)$ & $0.001^{2}$ \\
\hline
\end{tabular}

${ }^{1} \mathrm{UE}$ of inflexible differed statistically significantly of those whose psychological flexibility was low moderate $(\mathrm{p}=0.034)$, high moderate $(\mathrm{p}<0.001)$ or who were flexible $(\mathrm{p}<0.001)$ when tested using Tukey's multiple comparisons test; ${ }^{2} \mathrm{EE}$ of inflexible differed statistically significantly of those whose psychological flexibility was high moderate $(\mathrm{p}=0.013)$ or who were flexible $(\mathrm{p}<0.001)$ when tested using Tukey's multiple comparisons test.

Table 2. Psychological flexibility by occupational burnout.

\begin{tabular}{cccc}
\hline Psychological flexibility & No burnout $\mathrm{n}(\%)$ & Mild to serious burnout $\mathrm{n}(\%)$ & $\chi^{2}$-test $\mathrm{t}^{1}$ \\
\hline & $204(100)$ & $59(100)$ & $\mathrm{p}<0.001$ \\
Inflexible $\leq 48.4$ points & $37(18,1)$ & $30(50.8)$ & $20(33.9)$ \\
Moderate $48.5-61.6$ & $115(56.4)$ & $9(15.3)$ & \\
Flexible $\geq 61.7$ & $52(25.5)$ &
\end{tabular}

${ }^{1}$ The Pearson chi-square test was used to study differences between groups.

alone (Model 2, Figures 1 and 2).

The UE and EE of those without burnout who were psychologically inflexible [UE 47.7 (95\% CI 41.7, 53.8), EE $59.5(50.4,68.6)]$, and of those with burnout who were inflexible [UE41.6 $(34.9,48.4)$ EE 52.2 $(42.1,62.3)]$ or had moderate psychological flexibility [UE 48.9 (40.6, 57.1) EE $64.2(51.8,76.6)]$ were higher than the UE and EE of those without burnout who were flexible [UE 27.9 $(22.8,33.0)$ EE $34.6(26.9,42.3)]$ or had moderate flexibility [UE $30.7(27.2,34.1)$ EE $41.2(36.0,46.4)]$ or (Figures 1 and 2).

\section{DISCUSSION}

As far as we know, this is the first study to investigate the associations between occupational burnout, psychological flexibility and eating behaviour among middleaged working women. Diminished psychological flexibility associated with increased levels of EE and UE, and adjusting for flexibility diluted the association between occupational burnout and abnormal eating behaviour. Thus, flexibility can be a mediator between burnout and eating behaviour. However, the compound effect of psychological flexibility and occupational burnout was statistically significant, indicating that burnout and diminished psychological flexibility together increase the risk of high EE and UE. Thus, in addition to treatment of burnout among those who have high EE and UE and occupational burnout, improvement of their psychological flexibility could also prove highly beneficial.

The reason for high EE and UE among those with low psychological flexibility and among those with both low psychological flexibility and burnout may be an avoidant coping style to relieve difficult thoughts and feelings by eating [22,23]. Avoidant coping style and inflexibility may also be risk factors for job stress and occupational burnout [24]. However, occupational burnout may increase inflexibility because of the person's experiential avoidance of thoughts and feelings as a result of burnout. Certain thoughts and feelings may prevent these people acting according to their goals, e.g. making it hard to resist food cues [5]. The reinforcing effect of eating when stressed may also lead to learned behaviour in stressful situations which means that a person automatically eats when stressed. In addition, when a person is burned out and exhausted, they may be more vulnerable to acting in familiar manners and may not have the resources to change their behaviours, even when they wish to do so [20]. This may be why the combination of these two factors had the biggest effect on EE and UE in this study.

In this study, CR did not differ according to the level of psychological flexibility at baseline. Earlier studies have found that those whose restraint is flexible are more successful in weight loss than those whose restraint is rigid [25]. We did not study flexible and rigid restraint in this study, thus we could not determine whether the psychological flexibility of these two types of restraint eating differs. Future studies should take these two types of restraint into account to discover whether rigid restraint negatively associates with psychological flexibility and weight loss, and whether increasing psychological flexibility would help increase the flexible restraint related to weight loss. 


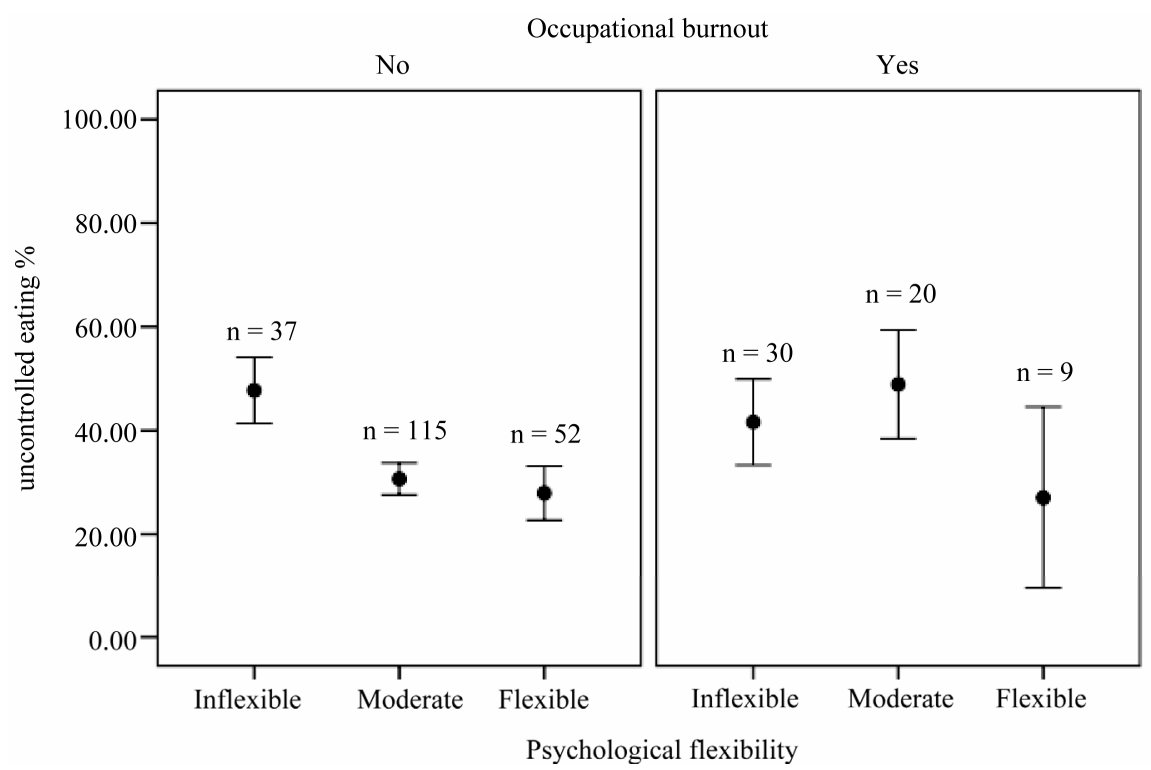

Figure 1. Uncontrolled eating mean (95\% confidence intervals) by psychological flexibility in burnout groups.

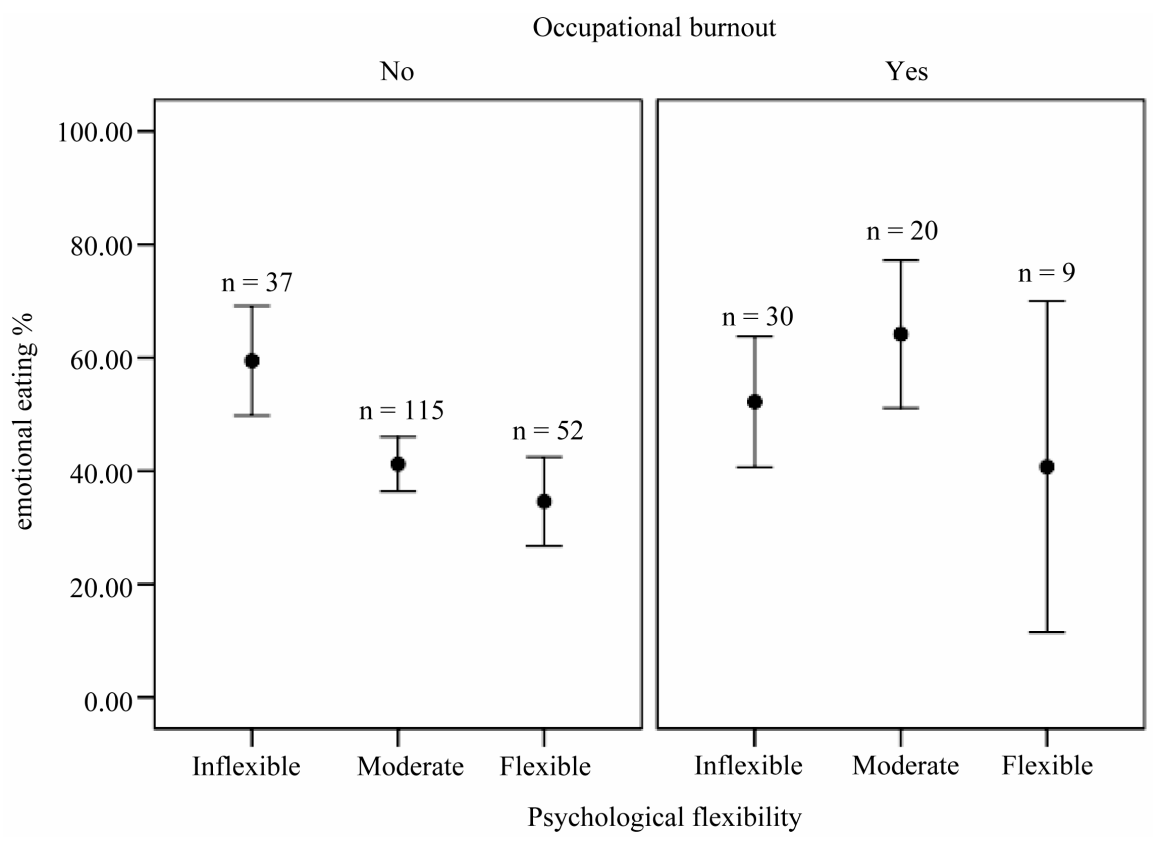

Figure 2. Emotional eating mean ( $95 \%$ confidence intervals) by psychological flexibility in burnout groups.

Because inflexibility was associated with high EE and UE, we can suggest that those who have high EE and UE could benefit from increasing psychological flexibility. Acceptance Commitment Therapy (ACT) might be helpful for increasing psychological flexibility $[4,26]$. In a review of ACT-based interventions, Ruiz (2012) suggested that participants with high experiential avoidance (i.e. inflexibility) respond differently to several experiential challenges, and acceptance- and mindfulness-based protocols are effective in improving the performance of the participants in different experiential challenges [7]. ACT is found to associate with increases in psychological flexibility among those with at least mild psychological distress [26]. Niemeier and colleagues (2012) found that among those who participated in acceptance-based behavioural intervention for weight loss, experiential avoidance, disinhibition and hunger decreased [27]. ACTbased group interventions have also improved participants eating disordered behaviours, body dissatisfaction, quality of life, acceptance of weight-related thoughts and 
feelings, and their distress tolerance and psychological flexibility $[28,29]$. Acceptance is associated positively with job satisfaction and job control, and negatively with mental ill-health [30], and increasing flexibility might also be helpful in preventing and decreasing symptoms of burnout [2,3,31]. Thus, increasing psychological flexibility might be helpful on many levels, and it might have positive consequences for the normalization of eating behaviour, weight management, and the reduction of occupational burnout.

\section{LIMITATIONS}

The limitations of the study were the small number of women suffering from burnout; this makes it difficult to generalize the results. The results can only be generalized to apply to middle-aged working women with rather good resources and mental health. To be eligible for the study, participants had to have evaluated their work ability score as between 7 and 9 on a scale of 0 to 10 , ten being their life time best work ability $[1,20]$. Thus, in spite of burnout, participants still felt that they had fairly good resources, or did not recognize their symptoms of burnout. It should also be noted that the range of those suffering from burnout (mild to serious) was quite wide, and only a few had serious burnout [1]. The participants also scored highly in psychological flexibility; only few had low psychological flexibility. However, occupational burnout and diminished psychological flexibility associated with high EE and UE, even if they were not very serious. Further studies with larger samples are needed to support these results.

\section{CONCLUSION}

This study showed that psychological inflexibility was associated with higher EE and UE, that psychological flexibility mediated the association between occupational burnout, EE and UE, and that psychological inflexibility together with occupational burnout associate with higher EE and UE. These cross-sectional associations should be confirmed in longitudinal studies. Intervention studies are also needed to evaluate whether increasing psychological flexibility, combined with dietary counselling, could be useful in normalizing the eating behaviour of those with psychological inflexibility and high EE and UE. In the case of serious burnout and reduced resources, burnout should be dealt with first, after which the target should be to normalize eating behaviour.

\section{REFERENCES}

[1] Nevanperä, N., Hopsu, L., Kuosma, E., Ukkola, O., Uitti, J. and Laitinen, J. (2012) Occupational burnout, eating behavior and weight among working women. American
Journal of Clinical Nutrition, 95, 934-943. doi:10.3945/ajen.111.014191

[2] Vilargada, R., Luoma, J.B., Hayes, S.C., Pistorello, J., Levin, M.E., Hildebrandt, M.J., Kohlenberg, B., Roget, N. A. and Bond, F. (2011) Burnout among the addiction counselling workforce: The differential roles of mindfulness and values-based process and work-site factors. Journal of Substance Abuse Treatment, 40, 323-335. doi:10.1016/j.jsat.2010.11.015

[3] Losa Iglesias, M.E., Becerro de Bengoa Vallejo, R. and Salvadores Fuentes, P. (2010) The relationship between experiential avoidance and burnout syndrome in critical care nurses: A cross-sectional questionnaire survey. International Journal of Nursing Studies, 47, 30-37. doi:10.1016/j.ijnurstu.2009.06.014

[4] Hayes, S.C., Luoma, J.B., Bond, F.W., Masuda, A. and Lillis, J. (2006) Acceptance and commitment therapy: Model, process and outcomes. Behaviour Research and Therapy, 44, 1-25. doi:10.1016/i.brat.2005.06.006

[5] Hayes, S.C. and Smith, S. (2005) Get out of your mind and into your life: The new acceptance \& commitment therapy. New Harbinger Publications, Oakland.

[6] Kingston, J., Clarke, S. and Remington, B. (2010) Experiential avoidance and problem behavior: A mediational analysis. Behavior Modification, 34, 145-163. doi:10.1177/0145445510362575

[7] Ruiz, F.J. (2010) A review of acceptance and commitment therapy (ACT) empirical evidence: Correlational, experimental psychopathology, component and outcome studies. International Journal of Psychology and Psychological Therapy, 10, 125-162.

[8] Karlsson, J., Persson, L.-O., Sjöström, L. and Sullivan, M. (2000) Psychometric properties and factor structure of the three-factor eating questionnaire (TFEQ) in obese men and women. Results from the Swedish Obese Subjects (SOS) study. International Journal of Obesity, 24, 17151725 .

[9] Dykes, J., Brunner, E.J., Martikainen, P.T. and Wardle, J. (2004) Socioeconomic gradient in body size and obesity among women: The role of dietary restraint, disinhibition and hunger in the Whitehall II study. International Journal of Obesity, 28, 262-268. doi:10.1038/sj.ijo.0802523

[10] Provencher, V., Drapeau, V., Tremblay, A., Després, J.-P. and Lemieux, S. (2003) Eating behaviors and indexes of body composition in men and women from the Québec family study. Obesity Research, 11, 783-792.

[11] Hays, N.P., Bathalon, G.P., McCrory, M.A., Roubenoff, R., Lipman, R. and Roberts, S.B. (2002) Eating behavior correlates of adult weight gain and obesity in healthy women aged 55-65 y. American Journal of Clinical Nutrition, 75, 476-483.

[12] Rutters, F., Nieuwenhuizen, A.G., Lemmens, S.G.T., Born, J.M. and Westerterp-Plantega, M.S. (2008) Acute stressrelated changes in eating in the absence of hunger. Behavior and Psychology, 17, 72-77. doi:10.1038/oby. 2008.493

[13] Keränen, A.-M., Savolainen, M.J., Reponen, A.H., Kujari, M.-L., Lindeman, S.M., Bloigu, R.S. and Laitinen, J. (2009) 
The effect of eating behavior on weight loss and maintenance during a lifestyle intervention. Preventive Medicine, 49, 32-38. doi:10.1016/i.ypmed.2009.04.011

[14] Konttinen, H., Haukkala, A., Sarlio-Lähteenkorva, S., Silventoinen, K. and Jousilahti, P. (2009) Eating styles, self-control and obesity indicators. The moderating role of obesity status and dieting history on restraint eating. Appetite, 53, 131-134. doi:10.1016/j.appet.2009.05.001

[15] Barraca, J. (2012) Mental control-From a third-wave behavior therapy perspective. International Journal of Clinical Health Psychology, 12, 109-121.

[16] Kearny, D.J., Milton, M.L., Malte, C.A., McDermott, K.A., Martinez, M. and Simpson, T.L. (2012) Participation in mindfulness-based stress reduction is not associated with emotional eating or uncontrolled eating. Nutrition Research, 32, 413-420. doi:10.1016/j.nutres.2012.05.008

[17] Lattimore, P., Fisher, N. and Malinowski, P. (2012) A cross-sectional investigation of trait disinhibition and its association with mindfulness and impulsitivity. Appetite, 56, 241-248. doi:10.1016/j.appet.2010.12.007

[18] Lillis, J., Hayes, S.C. and Levin, M.E. (2011) Binge eating and weight control: The role of experiential avoidance. Behavior Modification, 35, 252-264.

[19] Hopsu, L., Simonen, R., Halonen, J., Konttinen, J., Mattila, E., Lindholm, H. and Leino, T. (2010) Health promotion in occupational health care setting: Effects of an intensive health promotion: Nuadu study. Barents Newsletter on Occupational Health Safety, 13, 56-59.

[20] Näätänen, P., Aro, A., Matthiesen, S.B. and Salmela-Aro, K. (2003) Bergen burnout indicator 15. Edita Publishing Oy, Helsinki.

[21] Bond, F.W., Hayes, S.C., Baer, R.A., Carpenter, K.M., Guenole, N., Orcutt, H.K., Waltz, T. and Zettle, R.D. (2011) Preliminary psychometric properties of the acceptance and action questionnaire-II: A revised measure of psychological inflexibility and experiential avoidance. Behavior Therapy, 42, 676-688. doi:10.1016/j.beth.2011.03.007

[22] Lee, J.M., Greening, L. and Stoppelbein, L. (2007) The moderating effect of avoidant coping on restraint eaters' risk for disinhibited eating: Implications for dietary relapse prevention. Behaviour Research Therapy, 45, 2334-
2348. doi:10.1016/j.brat.2007.03.010

[23] Spoor, S.T.P., Bekker, M.H.J., Van Strien, T. and van Heck, G.L. (2007) Relations between negative affect, coping and emotional eating. Appetite, 48, 368-376. doi:10.1016/j.appet.2006.10.005

[24] Maslach, C., Schaufeli, W.B. and Leiter, M.P. (2001) Job burnout. Annual Review of Psychology, 52, 397-422.

[25] Westenhoefer, J., Stunkard, A.J. and Pudel, V. (1999) Validation of the flexible and rigid control dimensions of dietary restraint. International Journal of Eating Disorders, 26, 53-64.

[26] Fledderus, M., Bohlmeijer, E.T., Smit, F. and Westerhof, G.J. (2010) Mental health promotion as a new goal in public mental health care: A randomized controlled trial of an intervention enhancing psychological flexibility. American Journal of Public Health, 100, 2372-2378. doi:10.2105/AJPH.2010.196196

[27] Niemeier, H.M., Leahey, T., Reed, K.P., Brown, R.A. and Wing, R.R. (2012) An acceptance-based behavioral intervention for weight loss: A pilot study. Behavior Therapy, 43, 427-435. doi:10.1016/j.beth.2011.10.005

[28] Weineland, S., Arvidsson, D., Kakoulidis, T.P. and Dahl, J. (2012) Acceptance and commitment therapy for bariatric surgery patients, a pilot RCT. Obesity Research \& Clinical Practice, 6, e21-e30.

[29] Lillis, J., Hayes, S.C., Bunting, K.B. and Masuda, A. (2009) Teaching acceptance and mindfulness to improve the lives of the obese: A preliminary test of a theoretical model. Annals of Behavioral Medicine, 37, 58-69. doi:10.1007/s12160-009-9083-x

[30] Bond, F.W. and Bunce, D. (2003) The role of acceptance and job control in mental health, job satisfaction, and work performance. Journal of Applied Psychology, 88, 1057-1067.

[31] Brinkborg, H., Michanek, J., Hesser, H. and Berglung, G. (2011) Acceptance and commitment therapy for the treatment of stress among social workers: A randomized controlled trial. Behaviour Research and Therapy, 49 389-398. http://dx.doi.org.pc124152.oulu.fi:8080/10.1016/j.brat.20 $\underline{11.03 .009}$ 